\title{
APPLICATION OF THE METHOD OF FUNDAMENTAL SOLUTIONS TO THE ANALYSIS OF FULLY DEVELOPED LAMINAR FLOW AND HEAT TRANSFER
}

\author{
Krzysztof MrozeK \\ Poznan University of Technology, Institute of Mechanical Technology, Poznan, Poland \\ e-mail: krzysztof.mrozek@put.poznan.pl
}

\section{Magdalena MierzwiczaK}

Poznan University of Technology, Institute of Applied Mechanics, Poznań, Poland

e-mail: magdalena.mierzwiczak@wp.pl

\begin{abstract}
In this study, fully developed laminar flow and heat transfer in an internally longitudinally finned tube are investigated through application of the meshless method. The flow is assumed to be both hydrodynamically and thermally developed, with a uniform outside-the-wall temperature. The governing equations have been solved numerically by means of the method of fundamental solutions in combination with the method of particular solutions to obtain the velocity and temperature distributions. The advantage of the proposed approach is that it does not require mesh generation on the considered domain or its boundary, but uses only a cloud of arbitrarily located nodes. The results, comprising the friction factor as well as the Nusselt number, are presented for varied length values and fin numbers, as well as the thermal conductivity ratio between the tube and the flowing fluid. The results show that the heat transfer improves significantly if more fins are used.
\end{abstract}

Keywords: laminar flow, finned tube, heat transfer enhancement, method of fundamental solutions

\section{Introduction}

At present, many thermal engineering researchers are investigating new heat transfer enhancement methods between surfaces and the surrounding fluids. Heat transfer enhancement is of particular importance to the intensification of cooling of injection molds working in a cycle of dynamic temperature changes and equipped with cooling inserts. The aforesaid injections are the most important facet of the plastic injection molding process and affect the shape, aesthetics, technical properties and utility of compacts (Benitez-Rangel et al., 2010). One of the major problems encountered during the injection process is to ensure the most efficient and uniform heat transfer from the cooled material so as to avoid generation of excessive stress causing deformation of the molds. It should be emphasized that the cooling process can make up to $70 \%$ of the time cycle, and is one of the most important stages of the injection process. So far, the most common method of heat removal through application of cooling channels relies on conventional drilling. In order to improve the cooling efficiency, we propose the use of a finned cooling channel whose main task is to increase the active surface area of the heat exchange between the injection mold and the cooling fluid.

A comprehensive report on recent advances in heat transfer enhancements was presented by Siddique et al. (2010), while the classification of heat transfer enhancement techniques was documented by Bergles (1998). The mechanisms of enhancing heat transfer that require external power, dubbed active methods, comprise, for example, application of stirring in the fluid vessels or surface vibration, as presented by Nesis et al. (1994). The passive enhancement methods are 
those that do not require external power to sustain enhancement characteristics and rely on the use of: treated surfaces, rough surfaces, extended surfaces, displaced enhancement devices, swirl flow devices, coiled tubes, surface tension devices, additives for fluids and many others.

Most of the heat transfer augmentation methods presented in the literature employ fins. Laminar flow heat transfer in internally finned tubes is of particular importance in many engineering industries relying on the heating or cooling of viscous liquids or oils, and specifically including heating of the circulating fluid in solar collectors and heat transfer in heat exchangers. Internally finned tubes are commonly used in engineering applications as efficient means to improve convective heat transfer while maintaining a small size and low weight. To provide additional heat transfer surfaces, various types of internal fins are utilized. However, when an array of fins is used to enhance heat transfer, the presence of fins may increase the pressure drop in the tube and reduce the mass flow rate. For this reason, the prime engineering focus is to optimize the geometry of fins that will maximize the heat transfer rate under space and cost constraints.

Extensive work has been carried out by different researchers, e.g., Rout et al. (2012), to analyze a laminar heat exchanger with fins of various shapes and sizes. Experimental investigations show that the heat transfer characteristics and flow friction are greatly influenced by the fin spacing, size, and shape. Soliman and Feingold (1977) obtained an analytical solution for a fully developed laminar flow, encompassing an extensive range of fin parameters (varying height, width and number). The resulting equation of velocity distribution was rendered in the form of infinite series involving arbitrary constants evaluated by equating the velocity and its radial derivative at the boundary. In contrast, Soliman et al. (1980) presented numerical analysis of the momentum and energy equations using a finite difference approach. Along with dimensionless velocity, the authors determined the temperature field and defined the Nusselt number. Furthermore, the fully developed laminar flow and convective heat transfer in an internally finned tube heat exchanger were investigated numerically through application of an explicit finite-difference scheme by Tien et al. (2012). The authors conducted additional experiments in a closed-loop device to verify the numerical results. So far, the laminar flow heat transfer problem has mostly been resolved through utilization of the Finite Difference Method (FDM) providing the solution in a discrete form; both, the differential equation and the boundary conditions are fulfilled only in an approximate manner. The meshless Method of Fundamental Solutions (MFS) is free of the disadvantages of the above mentioned method. In MFS, the approximated solution is conveniently rendered as a continuous function with continuous derivatives. In this method, the governing equation is fulfilled exactly and the approximation lies in the fulfillment of the boundary conditions. For the homogenous differential problem of the maximum principle, the maximum error is achieved on the boundary and can be controlled by the appropriate value of the method parameters. The foundations of this dynamically developed meshless procedure were given in the 1960s by Kupradze and Aleksidze (1964). However, the modern, computerized version of the method was proposed a decade later by Mathon and Johnston (1977). The research conducted since then, and presented by Chen et al. (2008), allowed expanding its scope and successful application in solving the inhomogeneous differential equations, nonlinear problems, transient problems, or inverse problems. In recent years, it has become increasingly popular due to its simplicity of implementation. In such cases, the solution is approximated by linear combinations of fundamental solutions with singularities placed on a fictitious boundary lying outside the considered domain. MFS was successfully applied to resolve the potential flow problems by Johnston and Fairweather (1984), the Helmholtz problems by Tsai et al. (2009), the biharmonic equation by Karageorghis and Fairweather (1987), the elliptic boundary value problems by Karageorghis and Fairweather (1998), the Poisson equation by Golberg (1995), the Stokes flow problems by Alves and Silvestre (2004), and the elasticity problems by Tsai (2007). A comprehensive review of MFS was presented by Golberg and Chen (1999). 
This study considers two-dimensional heat conduction through fins with a fixed volume. Both, the velocity and temperature field values are determined by means of MFS and the Method of Particular Solutions (MPS). For the velocity field, the analytical solution could be obtained with a defined and acceptable accuracy. In contrast, the temperature field problem is tackled iteratively, using the Radial Basis Function (RBF) and monomials to determine the particular solution, and MFS to work out the homogenous solutions of each iterative step. It is assumed that both, the fins and the fluid flow, are subjected to constant wall temperature conditions. The parameters of thickness, length, and number of fins as well as the thermal conductivity ratio of the fin to the working fluid are varied to obtain the friction factor as well as the Nusselt number values in the internally finned tube.

\section{Analytical formulation}

Figure 1 shows the cross section of the internally finned tube considered in this paper. A variable number of straight fins are evenly distributed around the circumference of the tube. Due to geometric symmetry of the flow domain, as shown in Fig. 1, the solution to the governing equations is sought only for a half of the region between the center lines of two consecutive fins $\Omega_{f}^{*}$; i.e. between $\theta=0$ and $\theta=\gamma$.
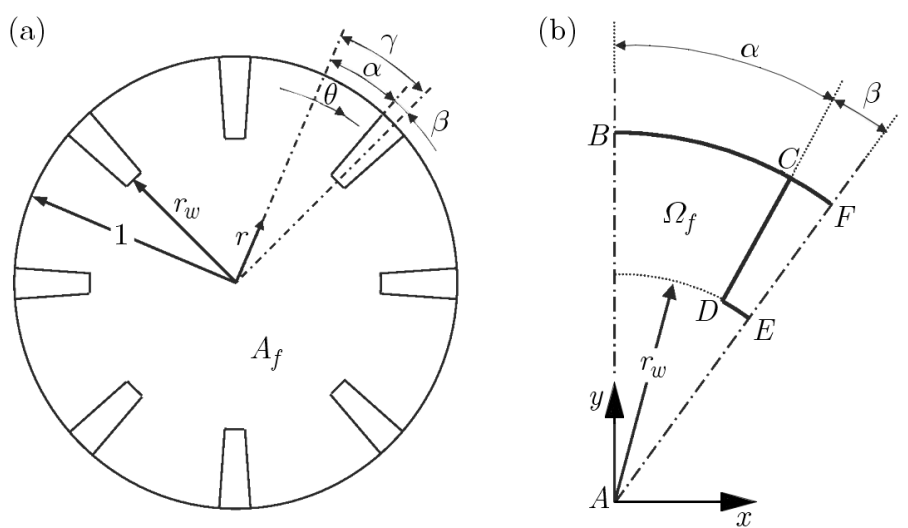

Fig. 1. Geometry of (a) the cross section of the tube and (b) a circular repeated part of the tube

\subsection{Determination of velocity}

This analysis is applicable to a steady, laminar, and fully developed flow with a uniform outside-the-wall temperature. Moreover, it is assumed that the fluid is Newtonian and has uniform properties, and the viscous dissipation within the fluid is neglected. On these assumptions, the momentum equation is reduced to

$$
\nabla^{2} w^{*}=\frac{1}{\mu} \frac{d p}{d z} \quad \text { in } \quad\left(x^{*}, y^{*}\right) \in \Omega_{f}^{*}
$$

where $w^{*}$ is the velocity along the tube; $\mu$ - dynamic viscosity; $d p / d z$ - gradient of pressure in the direction $z$. Using dimensionless variables, $r=r^{*} / r_{0}^{*}, x=x^{*} / r_{0}^{*}, y=y^{*} / r_{0}^{*}$, $w=w^{*} /\left[-(1 / \mu)(d p / d z) r_{0}^{*}\right]$, where $r_{0}^{*}$ is the inner radius of the tube, equation (2.1) can be written in a dimensionless form as

$$
\nabla^{2} w=-1 \quad \text { in } \quad(x, y) \in \Omega_{f}
$$

The boundary condition assumes the following form

$$
w=0 \quad \text { on } \quad(x, y) \in(\overline{B C} \cup \overline{C D} \cup \overline{D E})
$$


and

$$
\frac{\partial w}{\partial n}=0 \quad \text { on } \quad(x, y) \in(\overline{A B} \cup \overline{E A})
$$

and the dimensionless bulk velocity is obtained from the equation

$$
w_{b}=\frac{1}{A_{f}} \int_{A_{f}} w d A_{f}
$$

where $A_{f}=A_{f}^{*} / r_{0}^{* 2}$ is the dimensionless flow area of the tube; $A_{f}^{*}=\pi * r_{0}^{* 2}-M \beta\left(r_{0}^{* 2}-r_{w}^{* 2}\right)-$ the total flow area; $M$ - number of fins in the tube; $\beta$ - half of the angle subtended by one fin; $r_{w}=r_{w}^{*} / r_{0}^{*}-$ dimensionless radial coordinate at the tip of the fin, $L=L^{*} / r_{0}^{*}$-dimensionless fin height, $L^{*}-$ fin height.

The friction factor and the Reynolds number are defined as

$$
f=\frac{\pi^{2} \rho r_{0}^{* 5}}{\dot{m}^{2}}\left(-\frac{d p}{d z}\right) \quad \operatorname{Re}=\frac{2 \dot{m}}{\pi r_{0}^{*} \mu}
$$

where $\dot{m}=\rho A_{f}^{*} w_{b}^{*}$ is the mass flow rate of the fluid; $\rho$ - density of the fluid.

The product $f$ Re can be expressed in the dimensionless form

$$
f \operatorname{Re}=\frac{\pi}{A_{f}} \frac{2}{w_{b}}
$$

\subsection{Determination of temperature}

For a fully developed temperature profile, the dimensionless temperature $\left(T_{w}-T\right) /\left(T_{w}-T_{b}\right)$, where $T$ is the fluid temperature, $T_{b}$ is the bulk mean temperature, and $T_{w}$ is the tube-wall temperature, does not depend on $z$, that is

$$
\frac{\partial}{\partial z}\left(\frac{T_{w}-T}{T_{w}-T_{b}}\right)=0
$$

After some mathematical manipulations, (2.8) gives

$$
\frac{\partial T}{\partial z}=\frac{d T_{w}}{d z}-\frac{T_{w}-T}{T_{w}-T_{b}} \frac{d T_{w}}{d z}+\frac{T_{w}-T}{T_{w}-T_{b}} \frac{d T_{b}}{d z}
$$

Considering the constant-wall-temperature boundary condition, $d T_{w} / d z=0$, equation (2.9) can be reduced to

$$
\frac{\partial T}{\partial z}=\frac{T_{w}-T}{T_{w}-T_{b}} \frac{d T_{b}}{d z}
$$

If the flow is thermally developed and there is no axial conduction, the energy equation for the fluid flow takes the following form

$$
k_{f} \nabla^{2} T=\rho C_{p} w^{*} \frac{\partial T}{\partial z} \quad \text { in } \quad\left(x^{*}, y^{*}\right) \in \Omega_{f}^{*}
$$

where $k_{f}$ is the thermal conductivity of the fluid; $C_{p}$ - specific heat at constant pressure.

Introducing the dimensionless temperature of the fluid, $\Theta(x, y)=\left(T-T_{w}\right) /\left[q_{w}(z) r_{0}^{*} / k_{f}\right]$, where $q_{w}=Q /\left(2 \pi r_{0}^{*}\right)$ is the average heat flux at outer tube wall; $Q$ - total heat transfer rate at the solid-fluid interface, and employing (2.7) and (2.10) into equation (2.11), yields

$$
\nabla^{2} \Theta=\frac{\dot{m} C_{p}}{2 \pi q_{w} r_{0}^{*}} f \operatorname{Re} w \frac{\Theta}{\Theta_{b}} \frac{d T_{b}}{d z}
$$


For the energy balance of a small element $\Delta z$ in the axial direction of the tube, one obtains

$$
q_{w}\left(2 \pi r_{0}^{*}\right) \Delta z=\dot{m} C_{p}\left(\left.T_{b}\right|_{z+\Delta z}-\left.T_{b}\right|_{z}\right)
$$

As $\Delta z$ approaches zero, (2.13) can be simplified to

$$
\frac{d T_{b}}{d z}=\frac{q_{w} 2 \pi r_{0}^{*}}{\dot{m} C_{p}}
$$

Substituting (2.14) into (2.12) gives

$$
\nabla^{2} \Theta=f \operatorname{Re} w \frac{\Theta}{\Theta_{b}} \quad \text { in } \quad(x, y) \in \Omega_{f}
$$

and for the solid fin, the dimensionless energy equation becomes

$$
\nabla^{2} \Theta_{s}=0 \quad \text { in } \quad(x, y) \in \Omega_{s}
$$

The dimensionless boundary conditions for the fluid and the fin are

$$
\begin{aligned}
& \frac{\partial \Theta}{\partial n}=0 \quad \text { in } \quad(x, y) \in(\overline{A B} \cup \overline{E A}) \\
& \frac{\partial \Theta}{\partial n}=k \frac{\partial \Theta_{s}}{\partial n} \quad \wedge \quad \Theta=\Theta_{s} \in(x, y) \in(\overline{C D} \cup \overline{D E})
\end{aligned}
$$

and

$$
\begin{aligned}
& \frac{\partial \Theta_{s}}{\partial n}=0 \quad \text { in } \quad(x, y) \in \overline{E F} \\
& \Theta_{s}=0 \quad \text { in } \quad(x, y) \in \overline{F B} \\
& \Theta=0 \quad \text { in } \quad(x, y) \in \overline{B C}
\end{aligned}
$$

where $k=\beta k_{s} / k_{f}$ represents the ratio of thermal conductivity of the fin to the fluid, and $k_{s}$ is the thermal conductivity of the fin.

The dimensionless bulk mean temperature $\Theta_{b}$ and the Nusselt number $\mathrm{Nu}$ for the flow are derived as

$$
\Theta_{b}=\frac{\int_{A_{f}} w(x, y) \Theta(x, y) d A_{f}}{\int_{A_{f}} w(x, y) d A_{f}} \quad \mathrm{Nu}=\frac{2 r_{0}^{*} q_{w}(z)}{k_{f}\left(T_{w}-T_{b}\right)}=-\frac{2}{\Theta_{b}}
$$

\section{Numerical solution procedure}

To solve the boundary value problem for the velocity (2.2)-(2.4) and for the temperature (2.15)-(2.18), we propose to use the MFS.

The particular solution to (2.2) has the following form

$$
w^{p}=-\frac{1}{4}\left(x^{2}+y^{2}\right)
$$

and for the MFS, the homogenous solution can be represented as

$$
w^{h}=\sum_{n=1}^{N} c_{n} \ln r_{n}^{2}
$$


where $r_{n}=\sqrt{\left(x-\widetilde{x}_{n}\right)^{2}+\left(y-\widetilde{y}_{n}\right)^{2}}$ and $\left\{\left(\widetilde{x}_{n}, \widetilde{y}_{n}\right)\right\}_{n=1}^{N}$ are the coordinates of source points placed outside the considered region on a fictitious contour at a distance $s$ from the boundary (see Fig. 2). The unknown coefficients $\left\{c_{n}\right\}_{n=1}^{N}$ are obtained through fulfilling boundary conditions (2.3) and (2.4) in the collocation points $\left\{\left(x_{i}, y_{i}\right)\right\}_{i=1}^{M c}$

$$
\begin{aligned}
& \sum_{n=1}^{N} c_{n} \ln \left(\left(x_{i}-\widetilde{x}_{n}\right)^{2}+\left(y_{i}-\widetilde{y}_{n}\right)^{2}\right)=\frac{1}{4}\left(x_{i}^{2}+y_{i}^{2}\right) \quad \text { on } \quad\left(x_{i}, y_{i}\right) \in(\overline{B C} \cup \overline{C D} \cup \overline{D E}) \\
& \sum_{n=1}^{N} c_{n} \frac{\partial}{\partial n} \ln \left(\left(x_{i}-\widetilde{x}_{n}\right)^{2}+\left(y_{i}-\widetilde{y}_{n}\right)^{2}\right)=\frac{1}{2}\left(n x x_{i}+n y y_{i}\right) \quad \text { on } \quad\left(x_{i}, y_{i}\right) \in(\overline{A B} \cup \overline{E A})
\end{aligned}
$$

where $n=[n x, n y]$ is the unit outward normal vector at the boundary.

If the number of collocation points $M c$ is equal to the number of unknown coefficients, $\left\{c_{n}\right\}_{n=1}^{N} M c=N$, the system of algebraic equations (3.3) can be solved by the Gaussian elimination method. Otherwise, if $M c>N$, system (3.3) is overdetermined and is solved through application of the least squares approach.

The dimensionless velocity profile can be expressed as

$$
w=\sum_{n=1}^{N} c_{n} \ln r_{n}^{2}-\frac{1}{4}\left(x^{2}+y^{2}\right)
$$

Similarly, the solution to (2.16) can be written as a linear combination of fundamental solutions for the Laplace operator

$$
\Theta_{s}(x, y)=\sum_{n=1}^{N d} d_{n} \ln r_{d n}
$$

where $r_{d n}=\sqrt{\left(x-\widetilde{x}_{d n}\right)^{2}+\left(y-\widetilde{y}_{d n}\right)^{2}}$ and $\left\{\left(\widetilde{x}_{d n}, \widetilde{y}_{d n}\right)\right\}_{n=1}^{N d}$ are source points located around the fin area.

The homogenous solution to $(2.15)$ can be written as

$$
\Theta^{h}(x, y)=\sum_{n=1}^{N f} f_{n} \ln r_{f_{n}}
$$

where $r_{f n}=\sqrt{\left(x-\widetilde{x}_{f n}\right)^{2}+\left(y-\widetilde{y}_{f n}\right)^{2}}$ and $\left\{\left(\widetilde{x}_{f n}, \widetilde{y}_{f n}\right)\right\}_{n=1}^{N f}$ are source points located around the fluid area.

To obtain the particular solution to (2.15), we propose the use of RBF and monomials. The solution can be expressed as

$$
\Theta^{p}(x, y)=\sum_{m=1}^{M i} a_{m} \psi\left(r_{m}\right)+\sum_{j=1}^{K} b_{j} q_{j}(x, y)
$$

where $r_{m}=\sqrt{\left(x-\widehat{x}_{m}\right)^{2}+\left(y-\widehat{y}_{m}\right)^{2}}$ and $\left\{\left(\widehat{x}_{m}, \widehat{y}_{m}\right)\right\}_{m=1}^{M i}$ are interpolation points located in the fluid area, $\psi\left(r_{m}\right)$ is the particular solution of the RBS $\varphi\left(r_{m}\right)$ for the Laplace operator, and $q_{j}(x, y)$ is the particular solution of the monomials $p_{j}(x, y)$ for the Laplace operator

$$
\begin{array}{lr}
\nabla^{2} \psi\left(r_{m}\right)=\varphi\left(r_{m}\right) & m=1, \ldots, M i \\
\nabla^{2} q_{j}(x, y)=p_{j}(x, y) & j=1, \ldots, K
\end{array}
$$


The coefficients $\left\{a_{m}\right\}_{m=1}^{M i}$ and $\left\{b_{j}\right\}_{j=1}^{K}$ are calculated by interpolation of the right hand side of equation (2.15)

$$
\begin{array}{ll}
\sum_{m=1}^{M i} a_{m} \varphi\left(r_{m i}\right)+\sum_{j=1}^{K} b_{j} p_{j}\left(\widehat{x}_{i}, \widehat{y}_{i}\right) & =f \operatorname{Re} w\left(\widehat{x}_{i}, \widehat{y}_{i}\right) \frac{\Theta\left(\widehat{x}_{i}, \widehat{y}_{i}\right)}{\Theta_{b}} \quad i=1, \ldots, M i \\
\sum_{m=1}^{M} a_{m} p_{j}\left(\widehat{x}_{m}, \widehat{y}_{m}\right)=0 \quad j & =1, \ldots, K
\end{array}
$$

The coefficients $\left\{d_{n}\right\}_{n=1}^{N d}$ and $\left\{f_{n}\right\}_{n=1}^{N f}$ are obtained through fulfilling the boundary conditions (2.17) and (2.18) in the collocation points

$$
\begin{aligned}
& \sum_{n=1}^{N f} f_{n} \frac{\partial \ln r_{f_{n i}}}{\partial n}=-\sum_{m=1}^{M i} a_{m} \frac{\partial \psi\left(r_{m i}\right)}{\partial n}-\sum_{j=1}^{K} b_{j} \frac{\partial q_{j}\left(x_{i}, y_{i}\right)}{\partial n} \\
& \left\{\left(x_{i}, y_{i}\right)\right\}_{i=1}^{M 1+M 5} \in(\overline{A B} \cup \overline{E A}) \\
& \sum_{n=1}^{N f} f_{n} \ln r_{f_{n i}}=-\sum_{m=1}^{M i} a_{m} \psi\left(r_{m i}\right)-\sum_{j=1}^{K} b_{j} q_{j}\left(x_{i}, y_{i}\right) \quad\left\{\left(x_{i}, y_{i}\right)\right\}_{i=1}^{M 2} \in \overline{B C} \\
& \sum_{n=1}^{N f} f_{n} \frac{\partial \ln r_{f_{n i}}}{\partial n}+\sum_{n=1}^{N d} d_{n} \frac{\partial \ln r_{d_{n i}}}{\partial n}=-\sum_{m=1}^{M i} a_{m} \frac{\partial \psi\left(r_{m i}\right)}{\partial n}-\sum_{j=1}^{K} b_{j} \frac{\partial q_{j}\left(x_{i}, y_{i}\right)}{\partial n} \\
& \left\{\left(x_{i}, y_{i}\right)\right\}_{i=1}^{M 3+M 4} \in(\overline{C D} \cup \overline{D E}) \\
& \sum_{n=1}^{N f} f_{n} \ln r_{f_{n i}}+\sum_{n=1}^{N d} d_{n} \ln r_{d_{n i}}=-\sum_{m=1}^{M i} a_{m} \psi\left(r_{m i}\right)-\sum_{j=1}^{K} b_{j} q_{j}\left(x_{i}, y_{i}\right) \\
& \left\{\left(x_{i}, y_{i}\right)\right\}_{i=1}^{M 3+M 4} \in(\overline{C D} \cup \overline{D E}) \\
& \sum_{n=1}^{N d} d_{n} \frac{\partial \ln r_{d_{n i}}}{\partial n}=0 \quad\left\{\left(x_{i}, y_{i}\right)\right\}_{i=1}^{M 3} \in \overline{E F} \\
& \sum_{n=1}^{N d} d_{n} \ln r_{d_{n i}}=0 \quad\left\{\left(x_{i}, y_{i}\right)\right\}_{i=1}^{M 4} \in \overline{C F}
\end{aligned}
$$

The solution to (2.15) can be written as a sum

$$
\Theta(x, y)=\sum_{n=1}^{N f} f_{n} \ln r_{f_{n}}+\sum_{m=1}^{M i} a_{m} \psi\left(r_{m}\right)+\sum_{j=1}^{K} b_{j} q_{j}(x, y)
$$

Since the right hand side of governing equation (2.15) depends on the temperature $\Theta(x, y)$ as well as the bulk temperature value $\Theta_{b}$, we developed the following iterative procedure ascertaining its successful solution. First, we assumed uniform temperature conditions throughout the area $\Theta / \Theta_{b}=1$. The results are substituted into the right hand side of equation (2.15), which is then numerically solved for new values of $\Theta$. Based on these, a new value $\Theta_{b}$ is calculated and all results are again substituted into the right hand side of equation (2.15). The calculations are repeated until the values of $\Theta$ converge to acceptable tolerance values and carried out in accordance with the presented algorithm.

Step 0 Input of the data $M, \beta, k$, and $r_{w}$

Step 1 Determination of the optimal parameter $s$ characterized by the smallest error of the boundary value of temperature 
Step 2 Determination of the velocity value (2.2)-(2.4) by MFS (3.1)-(3.4)

Step 3 Computation of the bulk velocity $w_{b},(2.5)$, and the product $f$ Re, (2.7)

Step 4 Assumption that $\Theta^{0} / \Theta_{b}^{0}=1, i=1$

Step 5 Solution of interpolation problem (2.15) by RBF and monomials (3.9)

Step 6 Determination of temperature (2.15)-(2.18) by MFS (3.10) and (3.11)

Step 7 Calculation of the bulk mean temperature $\Theta_{b}^{i},(2.19)_{1}$, and the Nusselt number, $(2.19)_{2}$

Step 8 Convergence verification:

if $\delta \Theta=\left\|\Theta^{i}-\Theta^{i-1}\right\| \leqslant$ tol STOP,

else, take $i=i+1$ and go to Step $\mathbf{5}$

\section{Results and discussion}

In numerical experiments, as in those employing RBF, we use the thin-plate spline function

$$
\varphi\left(r_{m}\right)=r_{m}^{2} \ln r_{m}
$$

for which the particular solution for the Laplace operator has the form

$$
\psi\left(r_{m}\right)=\frac{r_{m}^{4} \ln r_{m}}{16}-\frac{r_{m}^{4}}{32}
$$

and six monomials presented in Table 1 .

Table 1. Monomial functions and their particular solutions

\begin{tabular}{|c|c|c|}
\hline$j$ & $p_{j}(x, y)$ & $q_{j}(x, y)$ \\
\hline \hline 1 & 1 & $\left(x^{2}+y^{2}\right) / 4$ \\
\hline 2 & $x$ & $x\left(x^{2}+y^{2}\right) / 8$ \\
\hline 3 & $y$ & $y\left(x^{2}+y^{2}\right) / 8$ \\
\hline 4 & $x y$ & $x y\left(x^{2}+y^{2}\right) / 12$ \\
\hline 5 & $x^{2}$ & $\left(x^{4}+x^{2} y^{2}-\frac{y^{4}}{6}\right) / 14$ \\
\hline 6 & $y^{2}$ & $\left(y^{4}+x^{2} y^{2}-\frac{x^{4}}{6}\right) / 14$ \\
\hline
\end{tabular}

An example of the distribution of collocation, the source and the interpolation points for the fluid area $\Omega_{f}$ and for the fin area $\Omega_{s}$ is shown in Fig. 2.

We calculate the number of the collocation and source points by means of the following formulas

$$
\begin{array}{ll}
M_{1}=111 & N_{1}=M_{1} / 3 \\
M_{2}=4 \alpha / \gamma M_{1} / M+1 & N_{2}=\left(N_{2}+N_{4}\right) / 3 \\
M_{3}=M_{1}\left(1-r_{w}\right)+1 & N_{3}=M_{3} / 3 \\
M_{4}=4 \beta / \gamma M_{1} / M+1 & N_{4}=M_{4} / 2 \\
M_{5}=M_{1} r_{w}+1 &
\end{array}
$$

To interpolate the right side function in (2.15), 378 evenly located points in the considered fluid area $(\widehat{x}, \widehat{y}) \in \Omega_{f}$ are used. The parameter $s$ proved to have a substantial impact on the 
(a)

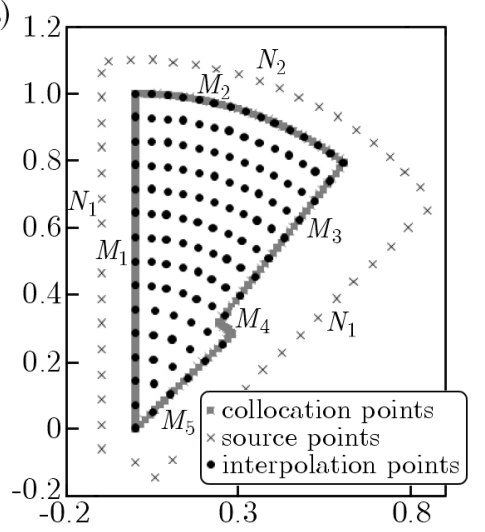

(b)

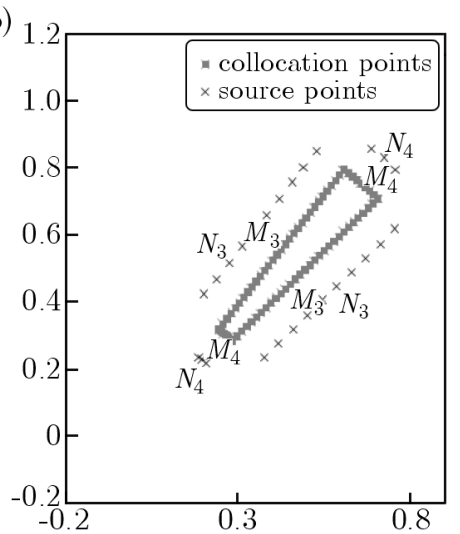

Fig. 2. Exemplary distribution of $\square c-$ collocation, $\times N$ - source, and $\bullet M i$ - interpolation points (a) in the fluid area and (b) in the solid fin area

accuracy of the presented method. Therefore, at the beginning of the calculation procedure, the optimal value of $s$ is determined, for which the maximum error of the boundary value of temperature in the control points is the lowest.

In order to verify the accuracy of the proposed algorithm, as the first test example, we have consitered a smooth tube without fins. The results of our calculations for different values of $\gamma$ are presented in Table 2 and are consistent with the literature, see e.g. Soliman et al. (1980). This allowed us, even at this early stage, to confirm the effectiveness of the proposed algorithm.

Table 2. Numerical results of the smooth tube investigation for different $\gamma$ values

\begin{tabular}{|c|c|c|c|c|c|}
\hline$M$ & $\gamma\left[^{\circ}\right]$ & $s_{O P T}$ & $f \mathrm{Re}$ & $\mathrm{Nu}$ & $\delta \Theta$ \\
\hline \hline 4 & 45 & 0.2121 & 16 & 3.6787 & $5.35 \mathrm{E}-06$ \\
\hline 8 & 22.5 & 0.1913 & 16 & 3.6773 & $5.47 \mathrm{E}-06$ \\
\hline 12 & 15 & 0.1812 & 16 & 3.6735 & $5.44 \mathrm{E}-06$ \\
\hline 16 & 11.25 & 0.1756 & 16 & 3.6707 & $5.41 \mathrm{E}-06$ \\
\hline 20 & 9 & 0.1721 & 16 & 3.6688 & $5.42 \mathrm{E}-06$ \\
\hline 24 & 7.5 & 0.1697 & 16 & 3.6676 & $5.41 \mathrm{E}-06$ \\
\hline 28 & 6.429 & 0.1679 & 16 & 3.6667 & $5.39 \mathrm{E}-06$ \\
\hline 32 & 5.625 & 0.1666 & 16 & 3.6662 & $5.32 \mathrm{E}-06$ \\
\hline
\end{tabular}

Furthermore, comparison between this study and previous work of Soliman and Feingold (1977) and Soliman et al. (1980) has been made to validate the postulated method. Figures $3 \mathrm{a}$ and $3 \mathrm{~b}$ show the comparison of $\mathrm{Nu}$ values obtained in this work and those of Soliman et al. (1980) for $k=1,5$, and 10 and for $M=4$ and 8. The maximum discrepancies in Nusselt numbers proved lower than $9 \%$ for $M=4$ and $12 \%$ for $M=8$. This is presumably due to the hereby assumed two-dimensional heat transfer, which differs from the one-dimensional fin conductance in the tube.

Further numerical results of $f$ Re for $\beta=3^{\circ}$, and for varying fin numbers and lengths, are presented in Fig. 4. The value of $f$ Re increases with the increase in $M$ for all values of $L$. The effect of $M$ is much more appreciable for longer fins.

The Nusselt number, defined by $(2.19)_{2}$, was used as a measure of the overall performance of any heat transfer surface; in other words, Nu reflects the influence of the internal finning on the overall heat transfer performance. The calculated values of $\mathrm{Nu}$ corresponding to the same value of the half of the angle of one fin, $\beta=3^{\circ}$, are listed in Table 3 for $k=\{1,5,10,100\}$ illustrating 

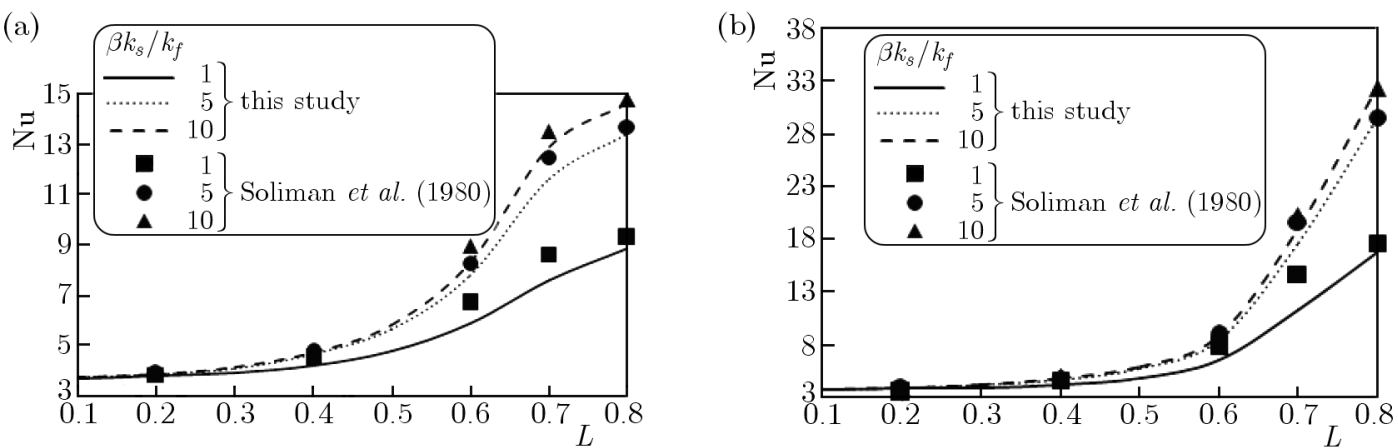

Fig. 3. Comparison of the results obtained in the present study with those presented by Soliman et al. (1980) for the solution of $f \operatorname{Re}$ for (a) $M=4$ and (b) $M=8$

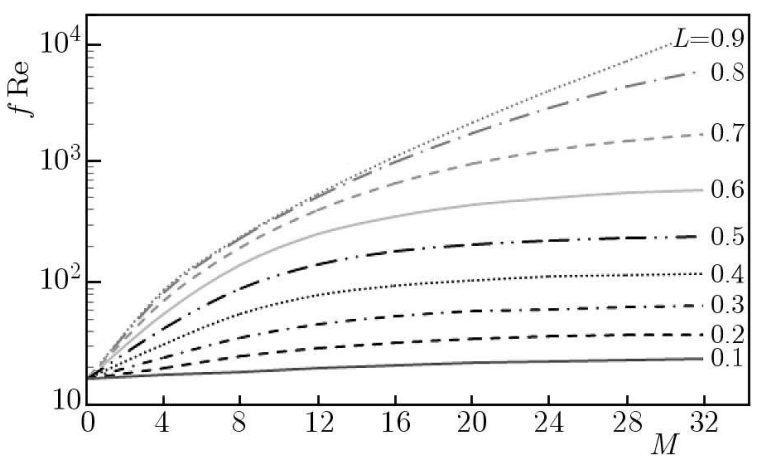

Fig. 4. The friction factor $f$ Re for $\beta=3^{\circ}$

different tube geometries. Obviously, the magnitude of heat transfer enhancement depends on $M, L$, and $k$. It can be observed that for any tube geometry, the value of $\mathrm{Nu}$ increases as $k$ increases. For a given value of $L$ and $k$, in most cases, the maximum value of $\mathrm{Nu}$ is obtained for the tube with eight fins $(M=8)$. The results depicted in Table 3 also show that the effect of $k$ is more visible for longer fins $(L \geqslant 0.7)$ when there are 4,8 , or 16 of them. For the tube with eight fins and 0.8 in length, for $k=1 \mathrm{Nu}=16.719$ and for $k=100$ the Nusselt number was more than two times greater $(\mathrm{Nu}=35.254)$. Comparing these results to those obtained for the smooth tube, the Nusselt number increases almost five times while, at the same time, the resistance increases more than 14 times.

A similar numerical experiment has been performed for constant values of the dimensionless flow area of the tube, $A_{f}=2.7$ (Table 4). For such a geometry of the tube, which changes the angle of the fins, $\beta=\left(\pi-A_{f}\right) /[M L(2-L)]$, the largest value of $\mathrm{Nu}$ is obtained for $M=16$ and $L=0.8$.

However, in this case, we deal with a high resistance value, $f \mathrm{Re}=671.92$. Considering the same example, for a fixed value of $\beta=3^{\circ}$ (Table 3), we observe that the resistance is much higher, $f \operatorname{Re}=975.96$ (since $A_{f}=2.337$ is smaller), and the value of the Nusselt number is smaller (although $\beta=3^{\circ}$ is larger). In the case of short fins $(L \leqslant 0.5)$, the largest reinforcement of the heat transfer $(\mathrm{Nu})$ is obtained for the tube with four fins (Table 4). This is advantageous, as in tubes with fewer fins the resistance $f$ Re is lower than in their multi-finned counterparts. From the numerical experiments, it appears that in order to intensify heat transfer, it is preferable to use tubes with slim fins, since, for $M=16$ and $L=0.8$, better results are obtained for $\beta=1.647$ and $A_{f}=2.7$ (Table 4) than for $\beta=3$ and $A_{f}=2.337$ (Table 3 ).

Figures 5 and 6 illustrate the effect of the angle $\beta$ of the fins of length $L=0.8$ on the enhancement of heat conduction, $\mathrm{Nu}$ and resistance to flow $f$ Re. The results show that $f$ Re and the width of the fins also play significant roles in enhancing the heat transfer. 
Table 3. Overall heat transfer results for $\beta=3^{\circ}$ and $A_{f}=\pi-M \beta L(2-L)$

\begin{tabular}{|c|c|c|c|c|c|c|c|c|c|c|c|}
\hline & & \multicolumn{2}{|c|}{$M=4$} & \multicolumn{2}{|c|}{$M=8$} & \multicolumn{2}{|c|}{$M=16$} & \multicolumn{2}{|c|}{$M=24)$} & \multicolumn{2}{|c|}{$M=32$} \\
\hline$L$ & $k$ & Re & $\mathrm{Nu}$ & $R e$ & $\mathrm{Nu}$ & $\mathrm{Re}$ & $\mathrm{Nu}$ & $\mathrm{Re}$ & $\mathrm{Nu}$ & $\mathrm{Re}$ & $\mathrm{Nu}$ \\
\hline \multirow{4}{*}{0.2} & 1 & \multirow{4}{*}{$\begin{array}{l}19.87 \\
A_{f}= \\
3.066\end{array}$} & 729 & \multirow{4}{*}{$\begin{array}{l}24.39 \\
A_{f}= \\
2.991\end{array}$} & 104 & \multirow{4}{*}{$\begin{array}{l}31.88 \\
A_{f}= \\
2.840\end{array}$} & & \multirow{4}{*}{$\begin{array}{l}35.74 \\
A_{f}= \\
2.689\end{array}$} & & \multirow{4}{*}{$\begin{array}{l}37.48 \\
A_{f}= \\
2.538\end{array}$} & .57 \\
\hline & 5 & & 3.793 & & 3.843 & & 3.739 & & 3.662 & & 3.66 \\
\hline & 10 & & 3.803 & & 3.852 & & 3.751 & & 3.695 & & 3.672 \\
\hline & 100 & & 3.812 & & 3.864 & & 3.773 & & 3.701 & & 3.67 \\
\hline & 1 & \multirow{4}{*}{$\begin{array}{l}31.47 \\
A_{f}= \\
3.008\end{array}$} & $\overline{172}$ & \multirow{4}{*}{$\begin{array}{l}55.50 \\
A_{f}= \\
2.874\end{array}$} & 4.100 & \multirow{4}{*}{$\begin{array}{l}93.89 \\
A_{f}= \\
2.605\end{array}$} & 3.647 & \multirow{4}{*}{$\begin{array}{c}110.85 \\
A_{f}= \\
2.337\end{array}$} & 3.480 & \multirow{4}{*}{$\begin{array}{c}117.90 \\
A_{f}= \\
2.069\end{array}$} & 3.48 \\
\hline & 5 & & 4.596 & & 4.617 & & 3.933 & & 3.696 & & 3.65 \\
\hline & 10 & & 4.685 & & 4.681 & & 3.981 & & 3.737 & & 3.68 \\
\hline & 100 & & 4.745 & & 4.758 & & 4.018 & & 3.771 & & $3.6 !$ \\
\hline & 1 & \multirow{4}{*}{$\begin{array}{l}42.04 \\
A_{f}= \\
2.985\end{array}$} & $\overline{737}$ & \multirow{4}{*}{$\begin{array}{l}89.79 \\
A_{f}= \\
2.827\end{array}$} & 4.737 & \multirow{4}{*}{$\begin{array}{c}179.13 \\
A_{f}= \\
2.513\end{array}$} & 3.801 & \multirow{4}{*}{$\begin{array}{c}223.23 \\
A_{f}= \\
2.199\end{array}$} & 3.435 & \multirow{4}{*}{$\begin{array}{c}242.01 \\
A_{f}= \\
1.885\end{array}$} & 3.392 \\
\hline & 5 & & 5.615 & & 5.663 & & 4.255 & & 3.748 & & 3.637 \\
\hline & 10 & & 5.796 & & 5.809 & & 4.319 & & 3.820 & & 3.678 \\
\hline & 100 & & 5.983 & & 5.960 & & 4.394 & & 3.884 & & 3.719 \\
\hline \multirow{4}{*}{.6} & 1 & \multirow{4}{*}{$\begin{array}{l}56.12 \\
A_{f}= \\
2.966\end{array}$} & 5.816 & \multirow{4}{*}{$\begin{array}{c}141.01 \\
A_{f}= \\
2.790\end{array}$} & 6.433 & \multirow{4}{*}{$\begin{array}{c}354.37 \\
A_{f}= \\
2.438\end{array}$} & 4.429 & \multirow{4}{*}{$\begin{array}{c}503.75 \\
A_{f}= \\
2.086\end{array}$} & 3.649 & \multirow{4}{*}{$\begin{array}{c}575.44 \\
A_{f}= \\
1.734\end{array}$} & 3.397 \\
\hline & 5 & & 7.754 & & 8.354 & & 5.158 & & 4.047 & & $\overline{3.741}$ \\
\hline & 10 & & 8.245 & & 8.715 & & 5.281 & & 4.121 & & 3.784 \\
\hline & 100 & & 8.776 & & 9.088 & & 5.400 & & 4.190 & & 3.82 \\
\hline \multirow{4}{*}{0.7} & 1 & & 7.541 & & .219 & & 7.005 & & 4.559 & & 3.567 \\
\hline & 5 & & 11.603 & & \begin{tabular}{|l|}
17.660 \\
\end{tabular} & & 8.654 & & 5.352 & & 4.025 \\
\hline & 10 & & 12.859 & & \begin{tabular}{|l|}
18.983 \\
\end{tabular} & & 8.942 & & 5.497 & & 4.092 \\
\hline & 100 & & 14.363 & & \begin{tabular}{|l|}
20.276 \\
\end{tabular} & & 9.243 & & 5.593 & & $4.14 !$ \\
\hline & 1 & & 8.845 & & \begin{tabular}{|l|}
16.719 \\
\end{tabular} & & \begin{tabular}{|l|}
20.063 \\
\end{tabular} & & 8.843 & & 5.000 \\
\hline & 5 & & 13.380 & & \begin{tabular}{|l|}
29.398 \\
\end{tabular} & & 28.323 & & 11.347 & & 5.858 \\
\hline & 10 & & 14.614 & & \begin{tabular}{|l|}
32.372 \\
\end{tabular} & & 29.694 & & 11.682 & & 5.975 \\
\hline & 10 & & .960 & & \begin{tabular}{|l|}
35.254 \\
\end{tabular} & & 31.272 & & 11. & & 6.06 \\
\hline
\end{tabular}

Further, it can be observed that $f$ Re varies linearly both for $M=4$ and $M=8$. However, when it comes to $\mathrm{Nu}$, for $M=4$ we have found some regularity, and for $M=8$ a significant increase in $\mathrm{Nu}$ has been obtained for $\beta=1.8$ (the result comparable to that obtained for $\beta=2.8$ ) and $\beta=2.0$ (the result comparable to that obtained for $\beta=3.0$ ). For both angles $\beta$ we observed enhancement of heat conduction while maintaining lower resistance $f$ Re.
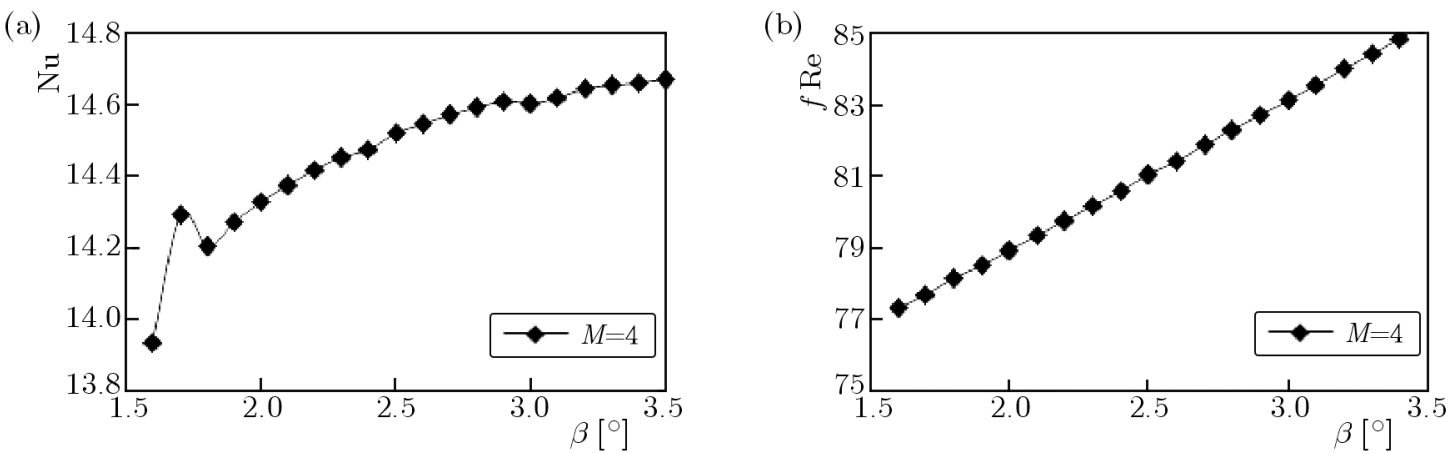

Fig. 5. The effects of the fin angle $\beta$ on the Nusselt number $\mathrm{Nu}$ and the product of the friction factor and the Reynolds number $f$ Re for $k=10, L=0.8$, and $M=4$ 
Table 4. Overall heat transfer results for $A_{f}=2.7$ and $\beta=\left(\pi-A_{f}\right) /[M L(2-L)]$

\begin{tabular}{|c|c|c|c|c|c|c|c|c|c|c|c|}
\hline & & \multicolumn{2}{|c|}{$M=4$} & \multicolumn{2}{|c|}{$M=8$} & \multicolumn{2}{|c|}{$M=16$} & \multicolumn{2}{|c|}{$M=24)$} & \multicolumn{2}{|c|}{$M=32$} \\
\hline$L$ & $k$ & Re & $\mathrm{Nu}$ & $R e$ & $\mathrm{Nu}$ & $\overline{\mathrm{Re}}$ & $\mathrm{Nu}$ & $\operatorname{Re}$ & $\mathrm{Nu}$ & $\operatorname{Re}$ & $\mathrm{Nu}$ \\
\hline \multirow{4}{*}{0.2} & 1 & \multirow{4}{*}{$\begin{array}{c}26.33 \\
\beta= \\
17.57\end{array}$} & 26 & \multirow{4}{*}{$\begin{array}{c}30.11 \\
\beta= \\
8.785\end{array}$} & & \multirow{4}{*}{$\begin{array}{c}34.12 \\
\beta= \\
4.393\end{array}$} & .597 & \multirow{4}{*}{$\begin{array}{c}35.46 \\
\beta= \\
2.928\end{array}$} & 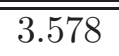 & \multirow{4}{*}{$\begin{array}{c}36.45 \\
\beta= \\
2.196\end{array}$} & \\
\hline & 5 & & 3.781 & & 3.761 & & 3.711 & & 3.682 & & 3.660 \\
\hline & 10 & & 3.822 & & 3.800 & & 3.717 & & 3.690 & & 3.675 \\
\hline & 100 & & 3.860 & & 3.827 & & 3.736 & & 3.696 & & 3.686 \\
\hline \multirow{4}{*}{0.4} & 1 & \multirow{4}{*}{$\begin{array}{c}41.53 \\
\beta= \\
9.883\end{array}$} & $\overline{992}$ & \multirow{4}{*}{$\begin{array}{c}63.82 \\
\beta= \\
4.942\end{array}$} & 3.987 & \multirow{4}{*}{$\begin{array}{c}90.81 \\
\beta= \\
2.471\end{array}$} & 3.710 & \multirow{4}{*}{$\begin{array}{c}101.76 \\
\beta= \\
1.647\end{array}$} & 3.597 & \multirow{4}{*}{$\begin{array}{c}107.48 \\
\beta= \\
1.235\end{array}$} & 3.571 \\
\hline & 5 & & 4.634 & & 4.484 & & 4.014 & & 3.795 & & 3.736 \\
\hline & 10 & & 4.767 & & 4.562 & & 4.055 & & 3.825 & & 3.775 \\
\hline & 100 & & 4.892 & & 4.634 & & 4.098 & & 3.876 & & 3.791 \\
\hline & 1 & \multirow{4}{*}{$\begin{array}{c}54.83 \\
\beta= \\
8.434\end{array}$} & 582 & \multirow{4}{*}{$\begin{array}{c}99.61 \\
\beta= \\
4.217\end{array}$} & 4.539 & \multirow{4}{*}{$\begin{array}{c}163.91 \\
\beta= \\
2.108\end{array}$} & 3.940 & \multirow{4}{*}{$\begin{array}{c}194.40 \\
\beta= \\
1.406\end{array}$} & 3.683 & \multirow{4}{*}{$\begin{array}{c}208.19 \\
\beta= \\
1.054\end{array}$} & 3.604 \\
\hline & 5 & & 5.758 & & 5.507 & & 4.410 & & 4.044 & & 3.920 \\
\hline & 10 & & 6.011 & & 5.655 & & 4.491 & & 4.096 & & 3.958 \\
\hline & 100 & & 6.278 & & 5.779 & & 4.585 & & 4.126 & & 3.991 \\
\hline \multirow{4}{*}{0.6} & 1 & \multirow{4}{*}{$\begin{array}{c}71.69 \\
\beta= \\
7.530\end{array}$} & 5.720 & \multirow{4}{*}{$\begin{array}{c}155.77 \\
\beta= \\
3.765\end{array}$} & 6.241 & \multirow{4}{*}{$\begin{array}{c}302.24 \\
\beta= \\
1.883\end{array}$} & 4.823 & \multirow{4}{*}{$\begin{array}{c}393.19 \\
\beta= \\
1.255\end{array}$} & 4.146 & \multirow{4}{*}{$\begin{array}{c}450.78 \\
\beta= \\
0.941\end{array}$} & 3.856 \\
\hline & 5 & & 8.128 & & 8.243 & & 5.664 & & 4.712 & & 4.260 \\
\hline & 10 & & 8.742 & & 8.551 & & 5.775 & & 4.784 & & 4.328 \\
\hline & 100 & & 9.473 & & 8.838 & & 5.964 & & 4.857 & & 4.367 \\
\hline \multirow{4}{*}{0.7} & 1 & & 7.505 & & 1.068 & & 8.221 & & 6.069 & & 5.241 \\
\hline & 5 & & 12.255 & & 17.470 & & 10.333 & & 7.254 & & 5.992 \\
\hline & 10 & & 13.775 & & 18.660 & & 10.842 & & 7.435 & & 6.039 \\
\hline & 100 & & 15.625 & & 19.885 & & 11.043 & & 7.497 & & 6.039 \\
\hline & 1 & & 8.730 & & 16.685 & & 24.696 & & 17.056 & & 12.921 \\
\hline & 5 & & 13.507 & & 29.539 & & 36.785 & & 21.135 & & 15.438 \\
\hline & 10 & & 14.785 & & 32.512 & & 38.967 & & 21.879 & & 15.737 \\
\hline & 100 & & 16.117 & & 35.340 & & 40.993 & & 22.791 & & 15.85 \\
\hline
\end{tabular}
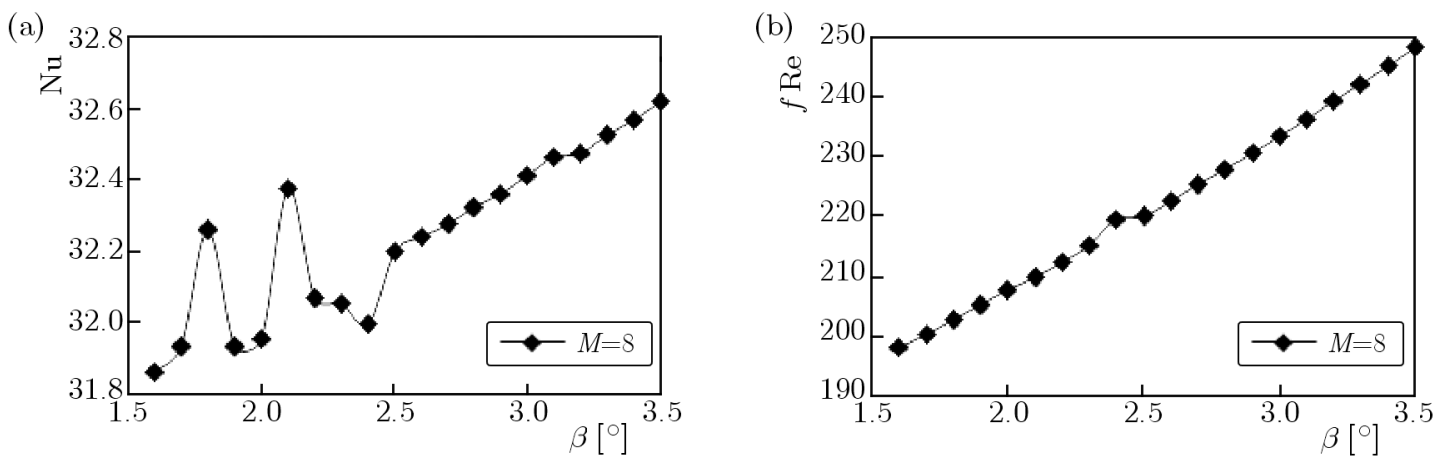

Fig. 6. The effects of the fin angle $\beta$ on the Nusselt number $\mathrm{Nu}$ and the product of the friction factor and the Reynolds number $f$ Re for $k=10, L=0.8$ and for $M=8$

\section{Conclusions}

In this paper, we have employed MFS with RBF to investigate a fully developed laminar flow and convective heat transfer in an internally finned tube. The presented method is very easy to implement, even in the case of highly challenging domains, because requires a cloud of points only. The hereby presented numerical results, pertaining to diverse experimental data, show that MFS is an accurate and reliable numerical technique generating solutions comparable with the literature. The proposed scheme is a competitive alternative to the existing 
methods of heat transfer investigation. The numerical analysis shows that the usage of finned cooling channels can not only contribute to the improvement of cooling effectiveness, but also, through proper fin placement, the enable manipulation of heat conduction in the mold. This finding is very significant from the technological point of view, as it will allow decreasing the stress strain of the mold, thereby improving its quality and mechanical properties.

Acknowledgments

The work has been supported by LIDER/006/143/L-5/13/NCBR/2014 Grant.

\section{References}

1. Alves C.J.S., Silvestre A.L., 2004, Density results using Stokeslets and a method of fundamental solutions for the Stokes equations, Engineering Analysis with Boundary Elements, 28, 10, $1245-1252$

2. Benitez-Rangel J.P., Trejo-Hernandez M., Morales-Hernandez L.A., Dominguez-Gonzalez A., 2010, Improvement of the injection mold process by using vibration through a mold accessory, Materials and Manufacturing Processes, 25, 7, 577-580

3. Bergles E., 1998., Handbook of Heat Transfer, McGraw-Hill, New York, NY, USA, 3rd edition

4. Chen C.S., Karageorghis A., Smyrlis T.S. (ED.), 2008, The Method of Fundamental Solutions - A Meshless Method. Dynamic Publishers, Inc., Atlanta

5. Golberg M.A., 1995, The method of fundamental solutions for Poisson's equation, Engineering Analysis with Boundary Elements, 16, 3, 205-213

6. Golberg M.A., Chen C.S., 1999, The method of fundamental solutions for potential, Helmholtz and diffusion problems, [In:] Boundary integral methods: numerical and mathematical aspects. Southampton: Computational Mechanics Publications, Golberg M.A. (Edit.), 103-176

7. Johnston R.L., Fairweather G., 1984, The method of fundamental solutions for problems in potential flow, Applied Mathematical Modelling, 8, 4, 265-270

8. Karageorghis A., Fairweather G., 1987, The method of fundamental solutions for the numerical solution of the biharmonic equation, Journal of Computational Physics, 69, 2, 434-59

9. Karageorghis A., Fairweather G., 1998, The method of fundamental solutions for elliptic boundary value problems, Advances in Computational Mathematics, 9, 69-95

10. Kupradze V.D., Aleksidze M.A., 1964, The method of functional equations for the approximate solution of certain boundary value problems, USSR Computational Mathematics and Mathematical Physics, 4, 4, 82-126

11. Mathon R., Johnston R.L., 1977, The approximate solution of elliptic boundary-value problems by fundamental solutions, SIAM Journal on Numerical Analysis, 14, 4, 638-650

12. Nesis E. I., Shatalov A. F., Karmatskit N. P., 1994, Dependence of the heat transfer coefficient on the vibration amplitude and frequency of a vertical thin heater, Journal of Engineering Physics and Thermophysics, 67, 1/2, 696-698

13. Rout S.K., Mishra D. P., Thatoi D. N., Acharya A. K., 2012, Numerical analysis of mixed convection through an internally finned tube, Advances in Mechanical Engineering, Vol.2012, Article ID 918342, p. 10

14. Siddique M., Khaled A.-R.A., Abdulhafiz N.I., Boukhary A.Y., 2010, Recent advances in heat transfer enhancements: a review report, International Journal of Chemical Engineering, vol. 2010, Article ID 106461, p. 28, doi:10.1155/2010/106461

15. Soliman H.M., Chau T.S., Trupp A.C., 1980, Analysis of laminar heat transfer in internally finned tubes with uniform outside wall temperature, Journal of Heat Transfer, 102, 598-604

16. Soliman H.M., Feingold A., 1977, Analysis of fully developed laminar flow in longitudinal internally finned tubes, Chemical Engineering Journal, 14, 119-128 
17. Tien W.-K., YeH R.-H., Hsiao, J.-C., 2012, Numerical analysis of laminar flow and heat transfer in internally finned tubes, Heat Transfer Engineering, 33, 11, 957-971

18. Tsai C.C., 2007, The method of fundamental solutions for three-dimensional elasto-static problems of transversely isotropic solids, Engineering Analysis with Boundary Elements, 31, 7, 586-594

19. Tsai C.C., Young D.L., Chiu C.L., Fan C.M., 2009, Numerical analysis of acoustic modes using the linear least squares method of fundamental solutions, The Journal of Sound and Vibration, 324, $3 / 5,1086-1100$

Manuscript received January 14, 2014; accepted for print March 11, 2014 